\title{
Review
}

\section{Cranberry anti-cancer compounds and their uptake and metabolism: An updated review}

\author{
Jeevan K. Prasain ${ }^{\mathrm{a}, *}$, Clinton Grubbs ${ }^{\mathrm{b}}$ and Stephen Barnes ${ }^{\mathrm{a}}$ \\ ${ }^{a}$ Department of Pharmacology and Toxicology, University of Alabama at Birmingham, Birmingham, AL, USA \\ ${ }^{\mathrm{b}}$ Department of Surgery, University of Alabama at Birmingham, Birmingham, AL, USA
}

Received 19 November 2018; accepted 6 January 2019

\begin{abstract}
Consumption of cranberry fruits or juice rich in polyphenols is associated with a wide range of potential health benefits. We and others have previously showed that cranberry juice concentrate and its phytochemicals, flavonols, anthocyanins and A-type proanthocyandins, may have potential to be chemopreventive agents. Although a number of cranberry constituents have been implicated in cancer prevention, our understanding about which metabolites are bio-available to reach target sites and thereby elicit cancer chemopreventive properties is still lacking. However, poor plasma bioavailability of cranberry constituents may be overcome by their potential interactions with gut microbiota by providing cancer prevention through induction of compositional and functional modifications of gut microbiota. Well-designed clinical trials evaluating metabolic and gut microbiome changes associated with cranberry consumption would provide useful information about the cancer patient's response to dietary intervention with cranberry constituents.
\end{abstract}

Keywords: Cranberry, polyphenols, chemoprevention, metabolism

\section{Introduction}

Cranberry (Vaccinium macrocarpon Ait. Ericaceae)- a fruit native to North America - offers a wide array of health benefits and has attracted much public interest as a functional food (whole, fortified or enriched food) with increasing scientific reports showing its potential health benefits [1]. It is widely believed that cranberry juice can help to treat and prevent urinary tract infections (UTIs) caused by E. coli [2]. Emerging evidence has also shown that cranberry juice and its phytochemicals have potential cancer chemopreventive effects both in vitro and in vivo [3-5].

Cranberry contains a diverse range of phytochemicals. Flavonols, proanthocyanidins (PACs), and anthocyanins are the major contributors to the observed anti-cancer properties of cranberry fruits or juice. Their underlying mechanisms of chemopreventive action may include induction of apoptosis in tumor cells [6], reduced ornithine decarboxylase activity [3], decreased expression of matrix metalloproteinase and anti-inflammatory activities [7]. In addition, other cancer preventive effects of cranberry have been attributed to modulating reactive oxygen

\footnotetext{
${ }^{*}$ Corresponding author: Jeevan Prasain, PhD, Department of Pharmacology \& Toxicology, MCLM 456, University of Alabama at Birmingham, 1918 University Boulevard, Birmingham, AL 35294, USA. Tel.: +1 205996 2612; Fax: +1 205 934 6944; E-mail: jprasain@ uab.edu.
} 
species (ROS) [8]. Cranberry may also be cancer preventive through a combination of additive and/or synergistic effects [9]. While a multitude of mechanisms has been proposed, clinical use of cranberry polyphenols as anti-cancer agents has not been approved due to several unresolved challenges.

For a safe and effective use of cranberry products, understanding the pharmacokinetic properties (absorption, distribution, metabolism and elimination or excretion, ADME) of a heterogeneous mixture or a pure compound isolated from cranberry is essential. However, a lack of authentic proanthocyanidin standards and sufficient amounts of these compounds for in vivo studies continue to be important challenges. In addition, safety evaluation is essential for a dietary supplement to be tested in humans.

With regard to cancer chemopreventive effects of cranberry and its active phytochemicals, there have been a number of excellent reviews [10-12]. The beneficial effects of cranberry constituents could be due to their metabolites presented at the target sites. In doing so, they may bind to target proteins, or modulate the activity of genes. Considering the importance of metabolic transformation of dietary polyphenols, in this review we attempt to provide an updated insight into the current stage of knowledge on anti-cancer compounds of cranberry and their uptake and metabolism in vitro and in vivo.

\section{Cranberry polyphenols with anti-cancer properties}

We previously demonstrated that cranberry juice may be effective in preventing urinary bladder carcinogenesis [5]. Our work on toxicological assessment of cranberry juice concentrate in Fischer-344 rats also concluded that exposures to cranberry juice concentrates do not pose a significant toxicology risk in rodents [5]. Recent research reports have further demonstrated that cranberry fruit can be a potential natural source for cancer prevention. Daily consumption of cranberry fruit powder (1500 mg daily for 30 days) lowered prostate specific antigen (PSA) in patients with prostate cancer [13]. Another study showed that dietary feeding of frozen cranberry may have potential to inhibit development of intestinal tumor in $\mathrm{APC}^{\mathrm{min} /+}$ mice [14].

Cranberry's efficacy against tumor development in vivo will depend largely on the bioavailability of its phytochemicals to the various tissues, but some of the cranberry components undergo rapid metabolism and elimination, indicating that they have poor bioavailability [15].

\subsection{Flavonols}

Flavonols are a class of flavonoids that have a 3-hydroxyflavone backbone. Quercetin, and myricetin (Fig. 1) are the main flavonols in cranberry and their levels vary among cultivars. They are mostly present in glycosylated forms. Less ripe fruits contain smaller amounts of flavonols in cranberry, compared to ripe ones [16]. Sugars often associated with these compounds are glucose, galactose, rhamnose, arabinose and glucuronic acid. The specificity of the sugar moiety and its attachment position to flavonol may play a role in the bioavailability in vivo [17].

\section{Quercetin}

Cranberry fruit contains glycosides (galactose, glucose, rhamnose and rutinose) of quercetin, with quercetin 3-galactoside being the predominant form. Quercetin has been shown to be a growth inhibitor against various cancers in vitro and in vivo [18-26]. Such inhibitory effects have been shown to depend on the form of quercetin. For example, compared to its rutinoside and rhamnoside, the aglycone quercetin has higher cytotoxic effects against human leukemia HL-60 cells [19]. Recent studies demonstrated quercetin's synergistic anti-cancer effects with 10-hydroxy camptothecin in MCF-7 cells, indicating its potential application of drug combination in cancer therapy [21]. While a number of reports demonstrate anti-cancer effects of quercetin, the underlying mechanism(s) 
<smiles>O=c1c(OC2O[C@H](CO)[C@@H](O)[C@H](O)[C@H]2O)c(-c2ccc(O)c(O)c2)oc2cc(O)cc(O)c12</smiles>

Quercetin 3-O-galactoside<smiles>COc1cc(C(=O)Oc2cc3c(O)cc(O)cc3cc2O)ccc1O</smiles>

Peonidin 3-O- galactoside<smiles>Oc1cc(O)c2c(c1)O[C@@]1(c3ccc(O)c(O)c3)Oc3cc(O)c4c(c3[C@@H]1O2)O[C@H](c1ccc(O)c(O)c1)[C@@H](O)C4</smiles>

Proanthocyanidin A2<smiles>O=c1c(O)c(-c2cc(O)c(O)c(O)c2)oc2cc(O)cc(O)c12</smiles>

Myricetin

Fig. 1. Structures of major cranberry polyphenols.

by which it exerts cancer chemoprevention are not yet understood [22]. Nonetheless, it interacts with multiple cellular targets influencing the activity of diverse signaling pathways [23].

Regarding the potential toxicity of quercetin in vivo, previous studies have shown that quercetin is not carcinogenic when rats were fed with 5.0\% (w/w) quercetin in diet [18, 24].

While quercetin intake is negatively correlated with the incidence of a number of cancers, its low bioavailability is a problem and combination therapy may provide solutions [25]. Uptake, metabolism, pharmacokinetics and biological effects of quercetins depend on their chemical structures and the biological systems being tested. Like other flavonoids, quercetin undergoes phase II metabolism, glucuronidation, methylation and sulfation. Quercetin-3-O- $\beta$-glucuronide (Fig. 2), the major metabolite of quercetin in circulation, may be hydrolyzed to the aglycone quercetin at target sites [27]. Phase II metabolites of quercetin and methylquercetin (quercetin sulfates, methylquercetin sulfate, quercetin diglucuronide, methylquercetin diglucuronide, methylquercetin glucuronide sulfate) were detected in the urine sample collected over $14 \mathrm{~h}$ after cranberry powder treatment in rats [15]. 
<smiles></smiles>

Quercetin 3-O-glucuronide<smiles>COc1cc(-c2oc3cc(O)cc(O)c3c(=O)c2O)ccc1O</smiles>

3'-methylquercetin<smiles>O=c1c(O)c(-c2ccc(O)c(OS(=O)(=O)O)c2)oc2cc(O)cc(O)c12</smiles>

Quercetin 3'-O- sulfate<smiles>NC(=O)CCC(=O)NC(CSc1c(-c2oc3cc(O)cc(O)c3c(=O)c2O)ccc(O)c1O)C(=O)NCC(=O)O</smiles>

Quercetin glutathione conjugate

Fig. 2. Representative structures of quercetin phase II metabolites.

Interestingly, in our studies, no free quercetin in plasma and urine was found: only low levels of methylated quercetin [15]. Intact quercetin 3-rhamnoside and quercetin-3-galactoside were detected in the urine treated with cranberry juice [15]. These results suggest that the glycosidic moiety plays a role in the absorption.

Although quercetin-3-O-glucoside is a minor flavonoid in cranberry, it is more bioavailable than its aglycone quercetin. The higher absorption of quercetin glucoside compared to aglycone is partly due to its absorption in the small intestine via the sodium-dependent glucose transporter (SGLT1) [28, 29]. Another possible mechanism is the hydrolysis of quercetin-3-glucoside by lactose phloridzin hydrolase present in the brush border membrane of the small intestine releasing the aglycone and sugar [30].

Cranberry constituents that are not absorbed in the small intestine may undergo metabolism by gut microbiota; however, the absorption ability of the colon is less efficient than that of the small intestine, albeit that transit time through the colon is much longer than through the small intestine. Inter-individual variability in gut microbiota and their interaction with host is another important issue related to polyphenol bioavailability and their potential health beneficial effects. An acute double-blind randomized control trial with cranberry juice showed that due to variations in the gut microbiome, inter-individual variation of plasma metabolites was broad and metabolite 
dependent [31]. Recent investigation on the relationship between quercetin metabolism, gut microbiota composition and dietary intake in elderly population indicate that specific fecal microbiota composition is correlated positively or negatively with quercetin concentration. It means metabolic fate of quercetin on the lower gut depends on the composition of microbiota [32]. Differences in phase II-enzyme polymorphism, in addition to the microbial composition of the gut microbiota, may be responsible for the inter-individual variation in the bioavailability of cranberry polyphenols [33].

In vivo beneficial effects of cranberry constituents depend on how they interact with target cells [34]. Understanding the intracellular metabolism of target compounds helps determine the underlying biological mechanism associated with cancer chemoprevention. In our lab we previously performed uptake and metabolism studies of quercetin and its metabolites in bladder cancer cells [35]. These studies showed that quercetin 3-O-glucuronide enters and remains intact but inactive in SW-780 cells. In contrast, quercetin 3-O-glucoside, quercetin, methylquercetin and myricetin exert cell growth inhibition [35].

Biological activities of polyphenols may be mediated by intracellular metabolites (including quinone/quinone methide species). In our studies, quercetin 3-O-galactoside and 3-O-glucoside differ from each other in terms of bladder cancer cell growth inhibition and cellular metabolism [35]. Compared to quercetin 3-O-glucoside, quercetin 3-O-galactoside (Fig. 2) undergoes less metabolic biotransformation (methylation, glucuronidation and quinone formation) and shows a weak cell growth inhibitory effect in SW-780 cells [35].

Glutathione is an abundant intracellular antioxidant and plays important roles in detoxifying of xenobiotics, including polyphenols. We and others have detected glutathionylated products of quercetin (Fig. 2) [34-36]. Quinone-like metabolites can be produced which may have role(s) in cell growth inhibition in the quercetintreated cells. We detected two peaks of quercetin glucuronides $(\mathrm{m} / \mathrm{z} 498)$ and one less mass unit $(\mathrm{m} / z$ 478) in the lysate of SW-780 bladder cancer cells treated with quercetin [35]. MS/MS of the precursor ions $\mathrm{m} / \mathrm{z} 479$ and 478 produced intense fragment ions $\mathrm{m} / z, 303$ and 302, respectively corresponding to aglycone quercetin $(\mathrm{m} / z, 303)$ and the proton stabilized quinone species $(\mathrm{m} / \mathrm{z}, 302)[34,35]$.

\section{Myricetin}

Myricetin (Fig. 1) is considered as a multi-targeted natural product with potential anti-migratory and antiinvasive effects based on in vitro cellular assays [37]. Recent preclinical studies showed that myricetin represses the malignant progression of prostate cancer [38]. It induces apoptosis in colon cancer cells by increasing the expression of nucleoside diphosphate kinase and other caspase-regulated apoptotic proteins [39]. Myricetin's potential anti-metastatic effects have been studied in a mouse model [40]. In this study, myricetin suppressed breast cancer metastasis to the lung, indicating its potential to be developed as a therapeutic agent for breast cancer. In our studies, myricetin showed potent cell growth inhibitory effects in bladder cancer cells, but low inhibitory activity in normal cells, indicating its selective cytotoxicity for cancer cells but not normal cells [35].

Not much is known about the uptake and metabolism of myricetin. Human urine contains myricetin-3galactoside and myricetin-arabinoside after ingestion of cranberry juice [41]. 3,5-Dihydroxyphenylacetic acid has been identified as its microbial metabolite in rats [42].

\section{Cranberry proanthocyanidins}

Cranberry fruits are a rich source of A-type proanthocyanidins and they may play a role in resistance to fruit rot [43]. Structurally, A-type PACs contain a double inter-flavanyl linkage compared to B-type PACs that have a single interflavanyl bond. Cranberry oligomers contain at least one A-type ether linkage $\left(\mathrm{C}_{2}-\mathrm{O}-\mathrm{C}_{7}\right)$ along with B-type linkages (Fig. 1). Their contents vary among cultivars growing in different regions. For example, Howes 
had the highest total proanthocyanidins (76-92 g kg-1) and Ben Lear had lower PAC content than others when eight cultivars were analyzed for PACs [43]. Processing cranberries also affects proanthocyanidin content [44].

A number of previous studies have shown that cranberry proanthocyanidins are potential chemopreventive or cytotoxic against cancer cells [12, 45-47]. Wang et al. demonstrated that quercetin aglycone and PAC DP-9 induce cytotoxicity and cell cycle arrest and increase cisplatin sensitivity in ovarian cancer cells [48]. Similarly, a cranberry proanthocyanidin (PAC-1A) has been shown to have chemotherapeutic potential to treat a broad spectrum of neuroblastomas, including highly malignant tumors that show resistance to cyclophosphamide by increasing its cellular uptake and retention [49]. Cranberry proanthocyanidins have been found to be among the most active constituents [12]. Kresty et al. showed potential preventive or therapeutic effects in human esophageal adenocarcinoma cell lines and esophageal tumor xenografts in athymic NU/NU mice [46]. Although PACs inhibit the growth of a number of cancer cells lines, proanthocyanidin A2 showed no potent growth inhibitory activities in bladder cancer cells [35].

Cranberry constituents including PAs may provide benefits on gastrointestinal cancer and UTIs, as well as systemic inflammatory activities mediated via the gut microbiome [50].

The low bioavailability of cranberry PAs in circulating plasma indicates that the major part of their uptake and metabolism takes place in the colon where the specific resident microbiota produce metabolites that can undergo further metabolism upon entering the systemic circulation.

\section{Anthocyanins}

Anthocyanins (the glycosides of anthocyanidins) are water-soluble flavonoids which impart visible pigmentation (absorb light at around $500 \mathrm{~nm}$ ) in fruits and vegetables. Daily intake of anthocyanins in the United States is in the range of $200 \mathrm{mg}$ which is about 9-fold higher than other flavonoids [51]. Cranberry contains glycosides of six aglycones - cyanidin, peonidin, malvidin, pelargonidin, delphinidin and petunidin - and the 3-O-galactoside of peonidin (Fig. 1). Cyanidins are the major anthocyanins (about 55\%) in cranberry [52]. Anthocyanins are potential cancer chemopreventive agents due to a broad range of activities, including anti-oxidant, anti-inflammatory and anti-mutagenic properties [53]. However, compared to anthocyanins, anthocyanidin aglycones show more potent cancer cell growth inhibitory activities in vitro [54]. Our lab previously examined growth inhibitory effects of the anthocyanins, cyanidin 3-O-glucoside, cyanidin 3-O-galactoside, and peonidin 3-glucoside, in bladder cancer cells. They all had weak activities, however [35].

Difference in the anti-cancer effects of anthocyanins is due to the different substituents on their B-ring; B-ring anthocyanins with ortho-dihydroxyphenyl substituents show the most potent activity [55]. There is an excellent updated review on effects of anthocyanins on the prevention and treatment of cancer by Lin et al., (2017) [53]. There has been considerable interest in cancer chemopreventive effects of anthocyanins because of their easily accessible source, low toxicity and large consumption via the diet [56].

Although anthocyanins are absorbed rapidly, overall, plasma bioavailability of anthocyanins is generally $<1 \%$ of the consumed amounts [57]. The food matrix also plays a role in determining their bioavailability [56]. We detected peonidin 3-O-galactoside and cyanidin-3-O-galactoside in rat urine after cranberry treatment [15]. Peonidin 3-O-galactoside was detected in urine within $24 \mathrm{~h}$ as the most abundant anthocyanin in healthy volunteers consuming $200 \mathrm{~mL}$ of cranberry juice; urinary output of anthocyanins reached a maximum between 3-6 h after ingestion [58]. In another pharmacokinetic study of cranberry anthocyanins in humans, galactosides, glucosides and arabinosides of cyanidin, peonidin and malvidin were detected in urine after consumption of cranberry juice [59]. Contrary to others' work, Kalt et al. (2014) detected a large number of anthocyanin phase II metabolites, especially aglycone glucuronides, following ingestion of blueberry juice in humans, indicating that anthocyanins are more bioavailable than previously suggested [60]. Another clinical study on metabolism of cyanidin-3-glucoside indicated that anthocyanins are metabolized to a structurally diverse range of metabolites (phase I, phase 2, degradants and bacterial metabolites) with dynamic kinetic profiles [61]. 


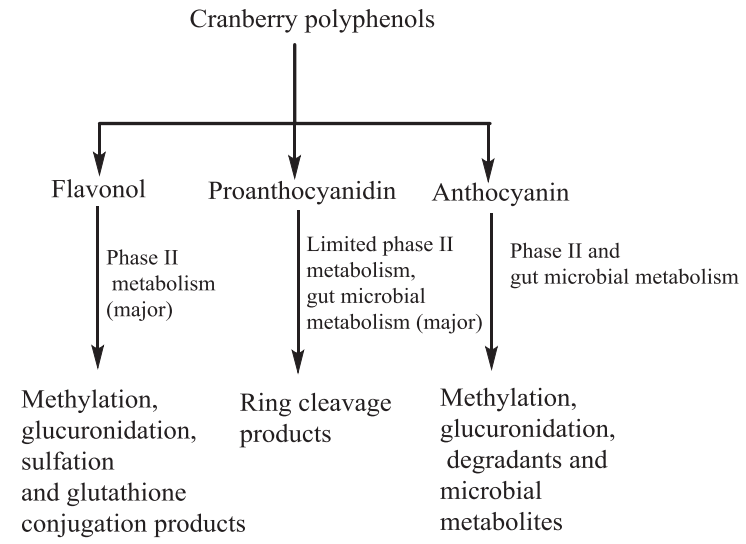

Fig. 3. Biotransformation of cranberry polyphenols in vitro and in vivo.

Because absorption of anthocyanins is an important issue if they are to be used as chemo-preventive agents, future studies focusing on enhancing their absorption is needed [62].

\section{Conclusions and future prospectus}

Although many preclinical studies have supported the cancer inhibitory potential of cranberries and their products, it is less clear whether the cancer chemo-preventive effects of cranberry are due to unmetabolized cranberry flavonoids or their upper intestinal and hepatic phase I and phase II metabolites. In addition, the role of specific gut bacteria in metabolism of cranberry polyphenols and the gut microbial metabolites that are absorbed into systemic circulation in exerting cancer chemoprevention remain to be elucidated, which are important issues in understanding the underlying mechanisms of action of cranberry. A summary of biotransformation of cranberry polyphenols is given in Fig. 3.

The bioavailability and activity of polyphenols found in fruits such as cranberry generally depend on not only in their content, but also on the extent to which they are metabolized by the gut microbiota, taken up into the blood and their hepatobiliary transport and metabolism by the liver. Although the gut microbial metabolism of dietary polyphenols is considered as an essential part of personalized nutrition approaches, whether gut microbiota regulate cranberry's chemo-preventive effects is largely unknown, partly because of the complex gut microbiota composition, which is further confounded by their interactions with the host. In addition, cranberry constituents may alter activities of fecal and host colonic mucosal enzymes, thereby altering cranberry and non-cranberry metabolites. Specific bacterial enzymatic activities such as glucosidases, esterases, demethylation, etc., may play important role in bio-activation or deactivation of cranberry metabolites. Therefore, future studies on effects of cranberry metabolites on the specific gut bacterial enzymatic activities are needed. Encouragingly, a recent randomized, double blind, cross-over feeding trial to evaluate cranberries' impact on gut microbiota has been reported [63]. These studies show that animal-based diets containing meat, dairy products and simple sugars altered gut microbiota composition to a less favorable profile and cranberries attenuate the impacts of animal diet.

Our current knowledge of gut microbial polyphenols metabolism generally is mainly based on interactions between single compounds and selected bacterial populations. To gain a comprehensive knowledge on interactions between cranberry constituents and the gut microbiome, metabolomics may provide functional endpoints associated with cancer chemo-prevention by cranberries. With regard to comprehensive analysis, recent high 
resolution mass spectrometry-based metabolomics studies show that cranberry juice consumption alters plasma levels of endogenous and hepatic or bacterial metabolites of cranberry in young women [64]. Such studies would help capture molecular signature that could lead to potential biomarker identification for cranberry chemoprevention. More specifically, by comparing metabolic profiles between cranberry-treated and untreated animals in specific animal models of cancers, we would know to what extent cranberry and non-cranberry metabolites are generated by colonic microbiota and their potential chemopreventive roles.

\section{Acknowledgments}

The authors have no acknowledgments.

\section{Funding}

The authors report no funding.

\section{Conflict of Interest}

The authors have no conflict of interest to report.

\section{References}

[1] Côté J, Caillet S, Doyon G, Sylvain JF, Lacroix M. Bioactive compounds in cranberries and their biological properties. Crit Rev Food Sci Nutr. 2010;50:666-79.

[2] Howell AB, Foxman B. Cranberry juice and adhesion of antibiotic-resistant uropathogens. JAMA. 2002;287:3082-3.

[3] Bomser J, Madhavi DL, Singletary K, Smith MA. In vitro anticancer activity of fruit extracts from Vaccinium species. Planta Med. 1996;62:212-6.

[4] Larocca LM, Giustacchini M, Maggiano N, Ranelletti FO, Piantelli M, Alcini E, Capelli A. Growth-inhibitory effect of quercetin and presence of type II estrogen binding sites in primary human transitional cell carcinomas. J Urol. 1994;152:1029-33.

[5] Prasain JK, Jones K, Moore R, Barnes S, Leahy M, Roderick R, Juliana MM, Grubbs CJ. Effect of cranberry juice concentrate on chemically-induced urinary bladder cancers. Oncol Rep. 2008;19:1565-70.

[6] Sun J, Hai Liu R. Cranberry phytochemical extracts induce cell cycle arrest and apoptosis in human MCF-7 breast cancer cells. Cancer Lett. 2006;241:124-34.

[7] Déziel BA, Patel K, Neto C, Gottschall-Pass K, Hurta RA. Proanthocyanidins from the American Cranberry (Vaccinium macrocarpon) inhibit matrix metalloproteinase-2 and matrix metalloproteinase-9 activity in human prostate cancer cells via alterations in multiple cellular signalling pathways. J Cell Biochem. 2010;111:742-54.

[8] Neto CC, Amoroso JW, Liberty AM. Anticancer activities of cranberry phytochemicals: An update. Mol Nutr Food Res. 2008;52(Suppl 1):S18-27.

[9] Seeram NP, Adams LS, Hardy ML, Heber D. Total Cranberry Extract versus Its Phytochemical Constituents: Antiproliferative and Synergistic Effects against Human Tumor Cell Lines. J Agric Food Chem. 2004;52:2512-7.

[10] Neto CC. Cranberry and its phytochemicals: A review of in vitro anticancer studies. J Nutr. 2007;137(1 Suppl):186S-193S.

[11] Neto CC. Cranberries: Ripe for more cancer research? J Sci Food Agric. 2011;91:2303-7.

[12] Weh KM, Clarke J, Kresty LA. Cranberries and Cancer: An Update of Preclinical Studies Evaluating the Cancer Inhibitory Potential of Cranberry and Cranberry Derived Constituents. Antioxidants (Basel). 2016;5(3).

[13] Student V, Vidlar A, Bouchal J, Vrbkova J, Kolar Z, Kral M, Kosina P, Vostalova J. Cranberry intervention in patients with prostate cancer prior to radical prostatectomy. Clinical, pathological and laboratory findings. Biomed Pap Med Fac Univ Palacky Olomouc Czech Repub. 2016;160:559-65. 
[14] Jin D, Liu T, Dong W, Zhang Y, Wang S, Xie R, Wang B, Cao H. Dietary feeding of freeze-dried whole cranberry inhibits intestinal tumor development in Apcmin/+mice. Oncotarget. 2017;8:97787-800.

[15] Rajbandhari R, Peng N, Moore R, Arabshahi A, Wyss JM, Barnes S, Prasain JK. Determination of cranberry phenolic metabolites in rats by liquid chromatography-tandem mass spectrometry. J Agric Food Chem. 2011;59:6682-8.

[16] Bilyk A, Sapers GM. Varietal differences in the quercetin, kaempferol, and myricetin contents of highbush blueberry, cranberry, and thornless blackberry fruits. J Agric Food Chem. 1986;34:585-8.

[17] Vvedenskaya IO, Rosen RT, Guido JE, Russell DJ, Mills KA, Vorsa N. Characterization of flavonols in cranberry (Vaccinium macrocarpon) powder. J Agric Food Chem. 2004;52:188-95.

[18] Hirose M, Fukushima S, Sakata T, Inui M, Ito N. Effect of quercetin on two-stage carcinogenesis of the rat urinary bladder. Cancer Lett. 1983;21:23-7.

[19] Shen SC, Chen YC, Hsu FL, Lee WR. Differential apoptosis-inducing effect of quercetin and its glycosides in human promyeloleukemic HL-60 cells by alternative activation of the caspase 3 cascade. J Cell Biochem. 2003;89:1044-55.

[20] Russo GL, Russo M, Spagnuolo C, Tedesco I, Bilotto S, Iannitti R, Palumbo R. Quercetin: A pleiotropic kinase inhibitor against cancer. Cancer Treat Res. 2014;159:185-205.

[21] Tang Q, Ji F, Wang J, Guo L, Li Y, Bao Y. Quercetin exerts synergetic anti-cancer activity with 10-hydroxy camptothecin. Eur J Pharm Sci. 2017;109:223-32.

[22] Chirumbolo S. Quercetin in cancer prevention and therapy. Integr Cancer Ther. 2013;12:97-102.

[23] Carvalho D, Paulino M, Polticelli F, Arredondo F, Williams RJ, Abin-Carriquiry JA. Structural evidence of quercetin multi-target bioactivity: A reverse virtual screening strategy. Eur J Pharm Sci. 2017;106:393-403.

[24] Okamoto T. Safety of quercetin for clinical application (Review). Int J Mol Med. 2005;16:275-8.

[25] Yang F, Song L, Wang H, Wang J, Xu Z, Xing N. Quercetin in prostate cancer: Chemotherapeutic and chemopreventive effects, mechanisms and clinical application potential. Oncol Rep. 2015;33:2659-68.

[26] Yang Y, Wang T, Chen D, Ma Q, Zheng Y, Liao S, Wang Y, Zhang J. Quercetin preferentially induces apoptosis in KRAS-mutant colorectal cancer cells via JNK signaling pathways. Cell Biol Int. 2018.

[27] Kawabata K, Mukai R, Ishisaka A. Quercetin and related polyphenols: New insights and implications for their bioactivity and bioavailability. Food Funct. 2015;6:1399-417.

[28] Hollman PCH, Devries JHM, Vanleeuwen SD, Mengelers MJB, Katan MB. Absorption of dietary quercetin glycosides and quercetin in healthy ileostomy volunteers. Am J Clin Nutr. 1995;62:1276-82.

[29] Hollman PCH, Buijsman MNCP, van Gameren Y, Cnossen PJ, de Vries JHM, Katan MB. The sugar moiety is a major determinant of the absorption of dietary flavonoid glycosides in man. Free Radic Res. 1999;31:569-73.

[30] Day AJ, Canada FJ, Diaz JC, et al. Dietary flavonoid and isoflavone glycosides are hydrolysed by the lactase site of lactase phlorizin hydrolase. FEBS Lett 2000;468:166-70.

[31] Feliciano RP, Mills CE, Istas G, Heiss C, Rodriguez-Mateos A. Absorption, Metabolism and Excretion of Cranberry (Poly)phenols in Humans: A Dose Response Study and Assessment of Inter-Individual Variability. Nutrients. 2017;9(3).

[32] Tamura M, Hoshi C, Kobori M, Takahashi S, Tomita J, Nishimura M, Nishihira J. Quercetin metabolism by fecal microbiota from healthy elderly human subjects. PLoS One. 2017;12:e0188271.

[33] McKay DL, Chen CY, Zampariello CA, Blumberg JB. Flavonoids and phenolic acids from cranberry juice are bioavailable and bioactive in healthy older adults. Food Chem. 2015;168:233-40.

[34] Spencer JP, Kuhnle GG, Williams RJ, Rice-Evans C. Intracellular metabolism and bioactivity of quercetin and its in vivo metabolites. Biochem J. 2003;372(Pt 1):173-81.

[35] Prasain JK, Rajbhandari R, Keeton AB, Gary A. Piazza GA, Barnes S. Metabolism and growth inhibitory activity of cranberry derived flavonoids in bladder cancer cells. Food Funct. 2016;7:4012-9.

[36] Awad HM, Boersma MG, Boeren S, Van Bladeren PJ, Vervoort J, Rietjens IM. Quenching of quercetin quinone/quinone methides by different thiolate scavengers: Stability and reversibility of conjugate formation. Chem Res Toxicol. 2003;16:822-31.

[37] Zhao HF, Wang G, Wu CP, Zhou XM, Wang J, Chen ZP, To ST, Li WP. A multi-targeted natural flavonoid myricetin suppresses lamellipodia and focal adhesions formation and impedes glioblastoma cell invasiveness and abnormal motility. CNS Neurol Disord Drug Targets. 2018;17:557-67.

[38] Ye C, Zhang C, Huang H, Yang B, Xiao G, Kong D, Tian Q, Song Q, Song Y, Tan H, Wang Y, Zhou T, Zi X, Sun Y. The natural compound myricetin effectively represses the malignant progression of prostate cancer by inhibiting PIM1 and disrupting the PIM1/CXCR4 interaction. Cell Physiol Biochem. 2018;48:1230-44.

[39] Lee JH, Choi YJ, Park SH, Nam MJ. Potential role of nucleoside diphosphate kinase in myricetin-induced selective apoptosis in colon cancer HCT-15 cells. Food Chem Toxicol. 2018;116(Pt B):315-22. 
[40] Ci Y, Zhang Y, Liu Y, Lu S, Cao J, Li H, Zhang J, Huang Z, Zhu X, Gao J, Han M. Myricetin suppresses breast cancer metastasis through down-regulating the activity of matrix metalloproteinase (MMP)-2/9. Phytother Res. 2018;32:1373-81.

[41] Wang Y, Singh AP, Nelson HN, Kaiser AJ, Reker NC, Hooks TL, Wilson T, Vorsa N. Urinary Clearance of Cranberry Flavonol Glycosides in Humans. J Agric Food Chem. 2016. [Epub ahead of print].

[42] Griffiths LA, Smith GE. Metabolism of myricetin and related compounds in the rat. Metabolite formation in vivo and by the intestinal microflora in vitro. Biochem J. 1972;130:141-51.

[43] Carpenter JL, Caruso FL, Tata A, Vorsa N, Neto CC. Variation in proanthocyanidin content and composition among commonly grown North American cranberry cultivars (Vaccinium macrocarpon). J Sci Food Agric. 2014;94:2738-45.

[44] Prior RL, Lazarus SA, Cao G, Muccitelli H, Hammerstone JF. Identification of procyanidins and anthocyanins in blueberries and cranberries (Vaccinium spp.) using high-performance liquid chromatography/mass spectrometry. J Agric Food Chem. 2001;49:1270-6.

[45] Weh KM, Aiyer HS, Howell AB, Kresty LA. Cranberry proanthocyanidins modulate reactive oxygen species in Barrett's and esophageal adenocarcinoma cell lines. J Berry Res. 2016;6:125-36.

[46] Kresty LA, Weh KM, Zeyzus-Johns B, Perez LN, Howell AB. Cranberry proanthocyanidins inhibit esophageal adenocarcinoma in vitro and in vivo through pleiotropic cell death induction and PI3K/AKT/mTOR inactivation. Oncotarget. 2015;6:33438-55.

[47] Kim KK, Singh AP, Singh RK, Demartino A, Brard L, Vorsa N, Lange TS, Moore RG. Anti-angiogenic activity of cranberry proanthocyanidins and cytotoxic properties in ovarian cancer cells. Int J Oncol. 2012;40:227-35.

[48] Wang Y, Han A, Chen E, Singh RK, Chichester CO, Moore RG, Singh AP, Vorsa N. The cranberry flavonoids PAC DP-9 and quercetin aglycone induce cytotoxicity and cell cycle arrest and increase cisplatin sensitivity in ovarian cancer cells. Int J Oncol. 2015;46:1924-34.

[49] Singh AP, Lange TS, Kim KK, Brard L, Horan T, Moore RG, Vorsa N, Singh RK. Purified cranberry proanthocyanidines (PAC-1A) cause pro-apoptotic signaling, ROS generation, cyclophosphamide retention and cytotoxicity in high-risk neuroblastoma cells. Int $\mathrm{J}$ Oncol. 2012;40:99-108.

[50] Blumberg JB, Basu A, Krueger CG, Lila MA, Neto CC, Novotny JA, Reed JD, Rodriguez-Mateos A, Toner CD. Impact of Cranberries on Gut Microbiota and Cardiometabolic Health: Proceedings of the Cranberry Health Research Conference 2015. Adv Nutr. 2016;7:759S-70S

[51] Wang LS, Stoner GD. Anthocyanins and their role in cancer prevention. Cancer Lett. 2008;269:281-90.

[52] Prior RL, Lazarus SA, Cao G, Muccitelli H, Hammerstone JF. Identification of procyanidins and anthocyanins in blueberries and cranberries (Vaccinium spp.) using high-performance liquid chromatography/mass spectrometry. J Agric Food Chem. 2001;49:1270-6.

[53] Lin BW, Gong CC, Song HF, Cui YY. Effects of anthocyanins on the prevention and treatment of cancer. Br J Pharmacol. 2017;174:1226-43.

[54] Zhang Y, Vareed SK, Nair MG. Human tumor cell growth inhibition by nontoxic anthocyanidins, the pigments in fruits and vegetables. Life Sci. 2005;76:1465-72.

[55] Hou DX, Kai K, Li JJ, Lin S, Terahara N, Wakamatsu M, Fujii M, Young MR, Colburn N. Anthocyanidins inhibit activator protein 1 activity and celltransformation: Structure-activity relationship and molecular mechanisms. Carcinogenesis. 2004;25:29-36.

[56] Yang M, Koo SI, Song WO, Chun OK. Food matrix affecting anthocyanin bioavailability: Review. Curr Med Chem. 2011;18:291-300.

[57] Manach C, Williamson G, Morand C, Scalbert A, Rémésy C. Bioavailability and bioefficacy of polyphenols in humans. I. Review of 97 bioavailability studies. Am J Clin Nutr. 2005;81(1 Suppl):230S-242S.

[58] Ohnishi R, Ito H, Kasajima N, Kaneda M, Kariyama R, Kumon H, Hatano T, Yoshida T. Urinary excretion of anthocyanins in humans after cranberry juice ingestion. Biosci Biotechnol Biochem. 2006;70:1681-7.

[59] Milbury PE, Vita JA, Blumberg JB. Anthocyanins are bioavailable in humans following an acute dose of cranberry juice. J Nutr. 2010;140:1099-104.

[60] Kalt W, Liu Y, McDonald JE, Vinqvist-Tymchuk MR, Fillmore SA. Anthocyanin metabolites are abundant and persistent in human urine. J Agric Food Chem. 2014;62:3926-34.

[61] de Ferrars RM, Czank C, Zhang Q, Botting NP, Kroon PA, Cassidy A, Kay CD. The pharmacokinetics of anthocyanins and their metabolites in humans. Br J Pharmacol. 2014;171:3268-82.

[62] Wang LS, Stoner GD. Anthocyanins and their role in cancer prevention. Cancer Lett. 2008;269:281-90.

[63] Prior RL, Lazarus SA, Rodríguez-Morató J, Matthan NR, Liu J, de la Torre R, Chen CO. Cranberries attenuate animal-based dietinduced changes in microbiota composition and functionality: A randomized crossover controlled feeding trial. J Nutr Biochem. 2018;62:76-86.

[64] Liu H, Garrett TJ, Su Z, Khoo C, Gu L. UHPLC-Q-Orbitrap-HRMS-based global metabolomics reveal metabolome modifications in plasma of young women after cranberry juice consumption. J Nutr Biochem. 2017;45:67-76. 